\title{
Realization of minimum number of rotational domains in heteroepitaxied $\mathrm{Si}(110)$ on $3 \mathrm{C}$ - $\operatorname{SiC}(001)$
}

Rami Khazaka, Marius Grundmann, Marc Portail, Philippe Vennéguès, Marcin Zielinski, Thierry Chassagne, Daniel Alquier, and Jean-François Michaud

Citation: Appl. Phys. Lett. 108, 011608 (2016); doi: 10.1063/1.4939692

View online: https://doi.org/10.1063/1.4939692

View Table of Contents: http://aip.scitation.org/toc/apl/108/1

Published by the American Institute of Physics

\section{Articles you may be interested in}

Turning the undesired voids in silicon into a tool: In-situ fabrication of free-standing 3C-SiC membranes

Applied Physics Letters 110, 081602 (2017); 10.1063/1.4977033

On the interplay between $\mathrm{Si}(110)$ epilayer atomic roughness and subsequent $3 \mathrm{C}-\mathrm{SiC}$ growth direction Journal of Applied Physics 120, 185306 (2016); 10.1063/1.4967741

Evidence of electrical activity of extended defects in 3C-SiC grown on $\mathrm{Si}$

Applied Physics Letters 96, 142104 (2010); 10.1063/1.3383233

Direct bandgap type-I GeSn/GeSn quantum well on a GeSn- and Ge- buffered Si substrate AIP Advances 8, 025104 (2018); 10.1063/1.5020035

Strain relaxation of germanium-tin (GeSn) fins

AIP Advances 8, 025111 (2018); 10.1063/1.5012559

Thermodynamic equilibration of the carbon vacancy in 4H-SiC: A lifetime limiting defect Journal of Applied Physics 122, 025701 (2017); 10.1063/1.4991815

\section{Conference Proceedings}




\title{
Realization of minimum number of rotational domains in heteroepitaxied $\mathrm{Si}(110)$ on $3 \mathrm{C}-\mathrm{SiC}(001)$
}

\author{
Rami Khazaka, ${ }^{1,2, a)}$ Marius Grundmann, ${ }^{3}$ Marc Portail, ${ }^{2}$ Philippe Vennéguès, ${ }^{2}$ \\ Marcin Zielinski, ${ }^{4}$ Thierry Chassagne, ${ }^{4}$ Daniel Alquier,${ }^{1}$ and Jean-François Michaud ${ }^{1}$ \\ ${ }^{1}$ Université François Rabelais, Tours, GREMAN, CNRS-UMR 7347, 16 rue Pierre et Marie Curie, \\ BP 7155, 37071 Tours Cedex 2, France \\ ${ }^{2}$ CRHEA, CNRS-UPR10, rue Bernard Gregory, 06560 Valbonne, France \\ ${ }^{3}$ Institut für Experimentelle Physik II, Fakultät für Physik und Geowissenschaften, Universität Leipzig, \\ Linnéstr. 5, 04103 Leipzig, Germany \\ ${ }^{4}$ NOVASiC, Savoie Technolac, Arche Bat 4, BP 267, 73375 Le Bourget du Lac Cedex, France
}

(Received 30 October 2015; accepted 28 December 2015; published online 8 January 2016)

\begin{abstract}
Structural and morphological characterization of a Si(110) film heteroepitaxied on 3C-SiC(001)/ $\mathrm{Si}(001)$ on-axis template by chemical vapor deposition has been performed. An antiphase domain (APD) free $3 \mathrm{C}$-SiC layer was used showing a roughness limited to $1 \mathrm{~nm}$. This leads to a smooth $\mathrm{Si}$ film with a roughness of only $3 \mathrm{~nm}$ for a film thickness of $400 \mathrm{~nm}$. The number of rotation domains in the $\mathrm{Si}(110)$ epilayer was found to be two on this APD-free 3C-SiC surface. This is attributed to the in-plane azimuthal misalignment of the mirror planes between the two involved materials. We prove that fundamentally no further reduction of the number of domains can be expected for the given substrate. We suggest the necessity to use off-axis substrates to eventually favor a single domain growth. (C) 2016 AIP Publishing LLC. [http://dx.doi.org/10.1063/1.4939692]
\end{abstract}

Silicon carbide $(\mathrm{SiC})$ is an interesting wide band-gap material due to the outstanding mechanical and electrical properties that this material has to offer. ${ }^{1,2}$ Compared to other $\mathrm{SiC}$ polytypes, cubic $\mathrm{SiC}$ (3C-SiC) can be heteroepitaxially grown on low-cost silicon ( $\mathrm{Si}$ ) substrates. ${ }^{3}$ In the last decades, 3C-SiC has been drawing attention for microsensing applications for Micro-Electro-Mechanical-Systems (MEMS) and Nano-EMS (NEMS). ${ }^{4-7}$ Our group investigated the benefit of growing a $\mathrm{Si}$ epilayer on top of $3 \mathrm{C}-\mathrm{SiC} /$ $\mathrm{Si}$ heterostructure to design MEMS devices benefiting from the properties of $3 \mathrm{C}-\mathrm{SiC} .^{8-10}$ Indeed, the difference in reactivity between $\mathrm{Si}$ and $3 \mathrm{C}-\mathrm{SiC}$ is advantageous for devices fabrication. ${ }^{11}$ This offers a high chemical selectivity between the two layers, where the Si epilayer can be used as a sacrificial layer and the 3C-SiC layer can serve as an etch-stop layer. However, 3C-SiC and $\mathrm{Si}$ feature a large lattice mismatch $(20 \%)$, a difference in thermal expansion coefficient $(8 \%)$ as well as different crystallographic structures (zinc blende vs. diamond, respectively). We shall note here that the diamond $\mathrm{Si}$ belongs to the $\mathrm{Fd} \overline{3} \mathrm{~m}$ space group and the cubic phase of $\mathrm{SiC}$ corresponds to the $\mathrm{T}^{2}{ }_{\mathrm{d}}-\mathrm{F} \overline{4} 3 \mathrm{~m}$ space group. These differences lead to a wide range of defects characterizing the $3 \mathrm{C}$-SiC film (stacking faults, micro-twins, and antiphase domains). The formation of antiphase domains (APDs) is one of the most serious issues limiting the development of $3 \mathrm{C}-\mathrm{SiC} / \mathrm{Si}$ devices. It has been shown that the APDs tend to annihilate with increasing 3C-SiC film thickness. ${ }^{12-14}$ Generally, for MEMS/NEMS devices, the heterointerface between the layers has to be smooth in order to avoid or limit the roughness transfer from one layer to the subsequent one, hence, avoid the process variability. Furthermore, the improvement of the material crystal quality

\footnotetext{
${ }^{\text {a) }}$ Author to whom correspondence should be addressed. Electronic mail: rami.khazaka@univ-tours.fr
}

strengthen its mechanical properties. Therefore, the 3C-SiC and the Si layers have to show a smooth surface with a good crystalline quality. It is well-known that the APDs in the $3 \mathrm{C}$ $\mathrm{SiC}$ layer drastically increase the roughness of the surface. ${ }^{15}$ Recently, we showed that the presence of APDs on the 3C$\mathrm{SiC}$ surface leads to domain formation in the Si layer rotated by $90^{\circ}$ around the growth direction. ${ }^{16}$ The presence of APDs on the $3 \mathrm{C}-\mathrm{SiC}$ surface is then expected to increase the roughness of the subsequent $\mathrm{Si}$ layer due to irregular domain boundaries which are detrimental for MEMS/NEMS structures. In addition, the heteroepitaxy of $\mathrm{Si}$ on $3 \mathrm{C}-\mathrm{SiC}$ is shown to occur in a columnar growth mode. ${ }^{16,17}$ This means that increasing the film thickness does not lead to any improvement of the crystal quality. The methods to improve the surface state and the final Si film quality are thus limited. Tuning the growth conditions can lead to an improvement of the crystalline quality and reduce the surface roughness to a certain extent. ${ }^{18}$ In fact, the best way to significantly ameliorate the final Si film quality and surface morphology at the same time is to completely eliminate the APDs on the $3 \mathrm{C}$ $\mathrm{SiC}$ surface. However, the difference in symmetry between two materials (3C-SiC $(001)$ and $\mathrm{Si}(110)$ in this case) leads to the formation of several domains in the grown layer.

The minimum number of domains that (must) form in the epilayer is fundamentally determined by the symmetry mismatch between the substrate and the epilayer. ${ }^{19,20}$ This minimum number falls from group theory and exists regardless of the kinetics, dynamics, and thermodynamics of the growth process. A large and representative number of reported epitaxial systems following group theory expectations has been corroborated in Ref. 19. Many substrate and epilayer combinations have a minimum number of domains larger than one. Various effects including approximately fulfilled symmetries can lead to additional domains. ${ }^{19}$ Such domains can also be generated in the layer depending on the 
growth conditions. It is fair to say, that any additional domain represents more grain types and increases disorder, typically leading to a degradation of the crystalline quality of the epilayer which is generally undesired. The attractive goal of decreasing the number of domains in the heteroepitaxied layer below the minimum limit for a given substrate orientation can only be achieved when the substrate symmetry is changed, typically reduced. For a desired substrate orientation, an off-cut of a few degrees can (i) provide the necessary break in symmetry, leading to a reduction of the number of domains, ${ }^{19}$ possibly down to one, and (ii) preserve the outof-plane epitaxial relationship and desired epilayer growth direction.

In this letter, the morphological and structural features of the $\mathrm{Si}(110)$ epilayer grown on APD-free 3C-SiC(001) surface were investigated. Relying on group theory, and due to the in-plane azimuthal misalignment of the mirror planes between the $3 \mathrm{C}-\mathrm{SiC}(001)$ film and the $\mathrm{Si}(110)$ epilayer, we will show that fundamentally the $\mathrm{Si}(110)$ layer must contain at least two domains.

The Si epilayer was heteroepitaxied on a $11 \mu \mathrm{m}$-thick $3 \mathrm{C}-\mathrm{SiC}(001) / \mathrm{Si}(001)$ template provided by NovaSiC. ${ }^{21}$ The 3C-SiC layer thickness was determined non-destructively by Fourier transform infrared spectroscopy (FTIR). The 3C-SiC layer is expected to exhibit a single domain. The growth of the Si layer was performed by chemical vapor deposition in a hot wall reactor by a mixture of $\mathrm{SiH}_{4}(1 \mathrm{sccm})$ and $\mathrm{H}_{2}(10$ slm) at $900^{\circ} \mathrm{C}$. The pressure was kept constant at 900 mbars during the growth process. Detailed information of the $\mathrm{Si}$ growth conditions can be found elsewhere. ${ }^{18}$ The Si film thickness was $0.4 \mu \mathrm{m}$ as measured by FTIR.

The surface morphology of both the 3C-SiC and Si layers was determined using tapping mode atomic force microscopy (AFM) (VEECO Dimension 3100). The crystal orientation determination of the $\mathrm{Si}$ film with respect to the $3 \mathrm{C}-\mathrm{SiC}$ film was performed with a Panalytical X'Pert Pro diffractometer using $\mathrm{Cu} \mathrm{K} \alpha$ radiation $(0.154 \mathrm{~nm})$ and by rotating the sample around the $\Phi$ axis at fixed $2 \theta$ and $\chi$ values.

Figure 1 shows large scale optical microscopy images of different 3C-SiC thicknesses heteroepitaxied under the same conditions and their corresponding AFM images. It is widely admitted that, in addition to the worsening of the crystal quality, the presence of APD domains on the $3 \mathrm{C}$-SiC surface increases its roughness due to the presence of irregular antiphase boundaries (APBs) between the two domains. ${ }^{12-14} \mathrm{~A}$ reduction of the surface roughness with the increase of the film thickness is obvious from the AFM images in Figure 1.

Figure 2 shows the amplitude derivative AFM images of the $3 \mathrm{C}-\mathrm{SiC}$ and the $\mathrm{Si}$ surfaces. It is well-known that the local 3C-SiC surface (i.e., APB-free) shows a smooth morphology characterized by atomic steps. In our case, the atomic steps can be observed on the single domain 3C-SiC surface (11- $\mu \mathrm{m}$ thick film), showing a rms roughness limited to $1 \mathrm{~nm}$ (Figure 2(a)). Since the domain formation in the $\mathrm{Si}$ epilayer is strictly linked to APDs on the $3 \mathrm{C}$-SiC surface, ${ }^{16}$ reducing the APB-density on the $3 \mathrm{C}-\mathrm{SiC}$ surface is expected to reduce the roughness of the top Si film. In Figure 2(b), the $\mathrm{Si}$ layer is shown to be smooth, the corresponding rms roughness of this surface is only $3 \mathrm{~nm}$, compared to a faceted surface obtained in other work ${ }^{17}$ and a roughness of above
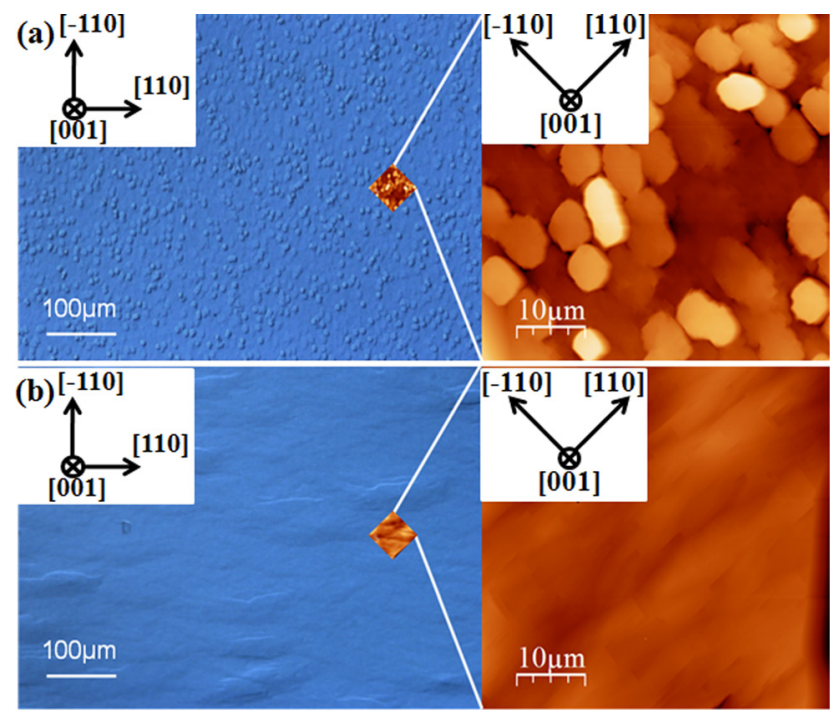

FIG. 1. Optical microscopy images (left) and atomic force microscopy images (right) $\left(50 \times 50 \mu \mathrm{m}^{2}\right)$ (a) of a $1.5 \mu \mathrm{m}$-thick 3C-SiC surface, (b) of a $11 \mu \mathrm{m}$-thick $3 \mathrm{C}$-SiC surface. The insets depict the AFM images adapted to the optical images scale.

$15 \mathrm{~nm}$ typically obtained using similar growth conditions on thin $(\sim 1 \mu \mathrm{m}) 3 \mathrm{C}$-SiC films. This low roughness value is desired for subsequent devices fabrication.

Structural study using XRD was performed on this heterostructure in order to investigate the domains repartition in the $\mathrm{Si}$ film and their epitaxial relation with the $3 \mathrm{C}-\mathrm{SiC}$ domain underneath it. The growth direction of the $3 \mathrm{C}-\mathrm{SiC}$ film is along the [001] axis while the top $\mathrm{Si}$ layer is oriented along the [110] direction as already reported in literature. ${ }^{16,17}$ The azimuthal $\Phi$-scan of the asymmetric (111) peaks (i.e., $2 \theta=35.69^{\circ}$, $\left.\chi=54.74^{\circ}\right)$ of $3 \mathrm{C}-\mathrm{SiC}(001)$ shows four peaks separated by $90^{\circ}$ in $\Phi$. These peaks are attributed to the diffraction of the $\{111\}$ set of planes in 3C-SiC(001) (cf. Fig. 3(a)).

For a defect-free $\mathrm{Si}(110)$ crystal, two (111) peaks separated by $180^{\circ}$ in $\Phi$ should appear at $\chi=35.26^{\circ}$. However, for a $\mathrm{Si}$ epilayer grown on $3 \mathrm{C}-\mathrm{SiC}(001)$ film, eight peaks are usually observed. ${ }^{16}$ The presence of these eight peaks is attributed to two reasons: (i) the presence of domains rotated by $90^{\circ}$ around the growth direction which increases the number of peaks from two to four and (ii) each domain have a twinned counterpart rising the number of peaks to eight. ${ }^{16,17}$ In Figure 3(b), the azimuthal $\Phi$-scan of the asymmetric (111) peaks of the $\operatorname{Si}(110)$ epilayer (i.e., $2 \theta=28.54^{\circ}$ and $\chi=35.26^{\circ}$ ) shows only four peaks. These four peaks are attributed to the
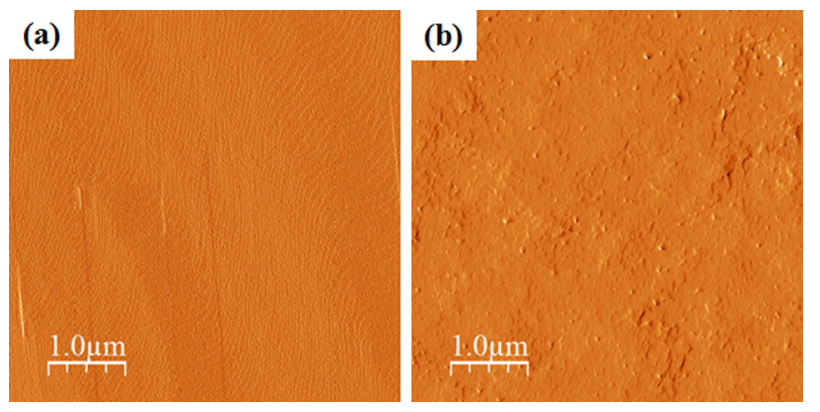

FIG. 2. Atomic force microscopy amplitude derivative images $\left(5 \times 5 \mu \mathrm{m}^{2}\right)$ (a) of an $11 \mu$ m-thick 3C-SiC surface (rms roughness limited to $1 \mathrm{~nm}$ ), (b) of the $\mathrm{Si}$ epilayer surface ( $\mathrm{rms}$ roughness $3 \mathrm{~nm}$ ) grown on $3 \mathrm{C}-\mathrm{SiC}$. 


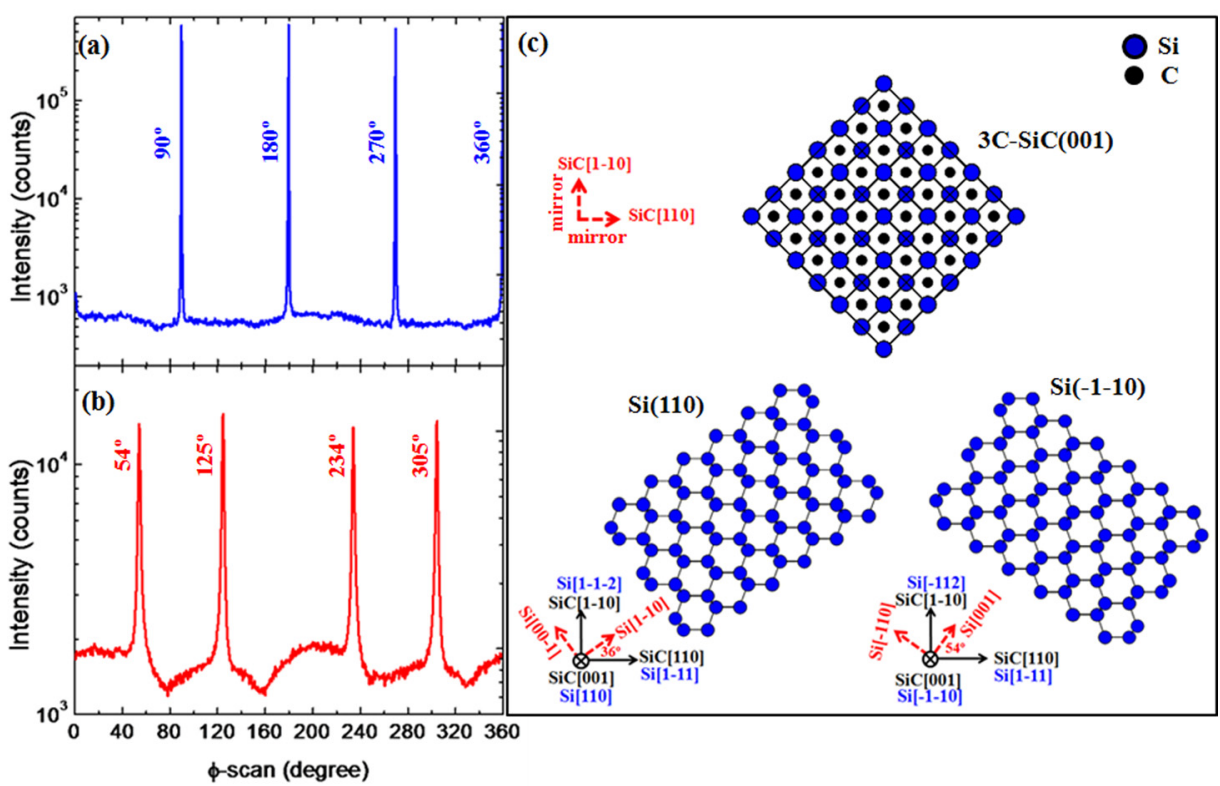

FIG. 3. Azimuthal XRD scans recorded along the asymmetric $\{111\}$ planes for (a) $3 \mathrm{C}-\mathrm{SiC}(001)$ film (i.e., $2 \theta=35.69^{\circ}$ and $\chi=54.74^{\circ}$ ) and (b) $\mathrm{Si}(110)$ film (i.e., $2 \theta=28.45^{\circ}$ and $\chi=35.26^{\circ}$ ). The 3C-SiC surface exhibits a single domain. (c) Atomic representation of the 3C-SiC surface and the two Si domains. The epitaxial relation between the 3C$\mathrm{SiC}$ film and the Si epilayer is indicated near the black arrows. The position of the mirror planes in each layer, respecting the given epitaxial relation, is indicated by dashed red arrows. presence of two domains having a twin relation with respect to each other (domains are grown along the [-1-10] direction instead of the [110] direction). Based on the peak positions of the Si epilayer relatively to those of the 3C-SiC film, the epitaxial relationship between the $3 \mathrm{C}-\mathrm{SiC}$ and the $\mathrm{Si}$ is thus: $3 \mathrm{C}-\mathrm{SiC}[001]$ parallel to $\mathrm{Si}[110], 3 \mathrm{C}-\mathrm{SiC}[110]$ parallel to $\mathrm{Si}$ [1-1-2], and 3C-SiC[1-10] parallel to Si[-11-1]. By defining surface lattice super cells, this orientation relationship shows a low lattice mismatch between the two materials. ${ }^{17}$ The difference in the lattice constant between $3 \times \mathrm{a}_{\mathrm{Si}[-112]}$ and $2 \times \mathrm{a}_{\mathrm{SiC}[1-10]}$ is $8 \%$, while this difference drops to only $1.9 \%$ between $\mathrm{a}_{\mathrm{Si}[-11-1]}$ and $\mathrm{a}_{\mathrm{SiC}[110]}$. An atomic illustration of the two domains in the $\mathrm{Si}(110)$ epilayer and the $3 \mathrm{C}-\mathrm{SiC}(001)$ film, taking into consideration the epitaxial relation between the two materials, is given in Fig. 3(c).

It has been shown that group theory can serve to predict the minimum number of rotational domains in heterostructures. A unified theory, based on the difference in symmetries between the two involved materials, has been developed by Grundmann et al. and it has been proved to be in agreement with many heterostructure systems. ${ }^{19,20}$ The formation of rotation domains in heterostructures strictly depends on the difference in symmetries between the film and the substrate. ${ }^{19,20}$ The minimum number of rotation domain in heteroepitaxy can be derived from the mismatch of the rotational symmetry between the substrate and the film. The substrate and the epilayer shall have a $C_{n}, C_{m}$ symmetry, respectively, with regard to the surface normal, where $\mathrm{n}$ and $\mathrm{m}$ are integers with possible values $\{1,2,3,4,6\}$. The rotational domains result from the mismatch of the rotational symmetries across the interface of the involved materials. In our case, a $180^{\circ}$ rotation around the $\mathrm{Si}[-11-1]$ axis was observed leading to the formation of two domains in the $\mathrm{Si}$ epilayer. This rotational domain was observed despite the same $2 \mathrm{~mm}$ two-dimensional point group symmetry of $3 \mathrm{C}-\mathrm{SiC}(001)$ and $\mathrm{Si}(110)$. We note that, for APD-free 3C-SiC(001) surface, the symmetry is $4 \mathrm{~mm}$ for the first monoatomic layer only while this symmetry is reduced to $2 \mathrm{~mm}$ for the infinite half space. ${ }^{19,22}$ At the early stage of $\mathrm{Si}$ nucleation, the Si adatoms interact with several monoatomic layers of the $3 \mathrm{C}-\mathrm{SiC}$ surface so that the $2 \mathrm{~mm}$ symmetry has to be considered for the $3 \mathrm{C}$ $\mathrm{SiC}(001)$. The $\mathrm{Si}(110)$ surface and half-space both show a $2 \mathrm{~mm}$ symmetry. A simple formula can be used to calculate the minimum expected number of domains $\left(\mathrm{N}_{\mathrm{RD}}\right)$ given in Ref. 20

$$
\mathrm{N}_{\mathrm{RD}}=\operatorname{lcm}(\mathrm{n}, \mathrm{m}) / \mathrm{m},
$$

where $1 \mathrm{~cm}$ denotes the least common multiple, $\mathrm{n}$ denotes the $\mathrm{C}_{\mathrm{n}}$ symmetry of the substrate, and $\mathrm{m}$ denotes the $\mathrm{C}_{\mathrm{m}}$ symmetry of the epilayer.

Taking into consideration the mentioned formula and the symmetry of the $3 \mathrm{C}-\mathrm{SiC}$ and the Si epilayer, the number of rotation domains in the Si epilayer must be equal to one. However, due to the aforementioned epitaxial relation between the $\mathrm{Si}$ epilayer and the $3 \mathrm{C}-\mathrm{SiC}$ film, the mirror symmetry planes of the $3 \mathrm{C}-\mathrm{SiC}(001)$ film (i.e., (110) and (1-10) planes) and the $\mathrm{Si}(110)$ epilayer (i.e., (001) and (1-10) planes) are misaligned. A rotation of $36^{\circ}$ exists between the $\mathrm{Si}(1-10)$ plane and the $3 \mathrm{C}-\mathrm{SiC}(110)$ plane (the $3 \mathrm{C}-\mathrm{SiC}(110)$ plane coincide with the $\mathrm{Si}(1-11)$ plane) as depicted in Fig. 3(c). Doubling the number of domains in the epilayer is generally induced by the presence of the substrate mirror symmetry, except when the mirror plane of the substrate and that of the epilayer are aligned. ${ }^{19}$ In the case of misalignment, the mirror operation of the substrate is no longer mirror symmetry for the epilayer. Increasing the number of domains by a factor of two has been observed in the case of gallium nitride $(\mathrm{GaN})$ on germanium $(\mathrm{Ge})$, where the $\mathrm{GaN}$ lattice has been observed to be rotated by $4^{\circ}$ compared to that of $\mathrm{Ge}^{23}$ The Ge mirror plane generated the presence of two $\mathrm{GaN}$ domains rotated by $\pm 4^{\circ}$ around the mirror plane (i.e., $8^{\circ}$ compared to each other). In our case, an angle of $36^{\circ}$ exists between the mirror symmetries of the $3 \mathrm{C}-\mathrm{SiC}(001)$ and the $\mathrm{Si}(110)$. Thus, the mirror symmetry of the $3 \mathrm{C}-\mathrm{SiC}$ surface allows the equivalent nucleation of $\mathrm{Si}$ domains rotated by $\pm 36^{\circ}$. These two domains have the same probability of presence and expansion. This is confirmed by the nearly identical intensities of the twinned domain compared to that of the main domain as 
attested by the XRD azimuthal $\Phi$-scan (Fig. 3(b)). This consideration highlights that, since the nucleation, the two domains have an equal probability of existence. Thus, the presence of the second domain in the Si layer is rather attributed to the angular misalignment of the mirror planes between the two materials than to the low formation energy of $\mu$-twins in the Si layer.

The surface reconstruction has been shown to switch from $(n \times m)$ to $(m \times n)$, where $n, m=[1,2,3, \ldots]$, between two antiphased domains on the $3 \mathrm{C}-\mathrm{SiC}$ surface. ${ }^{24}$ Thus, the surface can be treated as two local surfaces showing a $2 \mathrm{~mm}$ symmetry each, rotated by $90^{\circ}$ around the growth direction. Therefore, the number of expected domains increases to four in the $\mathrm{Si}$ epilayer as previously reported for the $\mathrm{Si}$ layer heteroepitaxied on $3 \mathrm{C}$-SiC. ${ }^{16}$

In the case of APD-free 3C-SiC(001) layer, the minimum number of domains that can be obtained in the Si epilayer is equal to two. This underlines that the growth was performed under the optimized conditions. Since these two $\mathrm{Si}$ domains are equally energetically/crystallographically favorable, further improvement seems to be impossible under our conditions. However, breaking the mirror symmetry of the $3 \mathrm{C}-\mathrm{SiC}$ by using a specific off-cut is expected to favor eventually the growth of only one domain at the expenses of the second domain.

In summary, we reported the growth of a Si epilayer on an APD-free 3C-SiC(001) surface. The surface morphology of the Si film shows an rms roughness of only $3 \mathrm{~nm}$. The APD-free $3 \mathrm{C}-\mathrm{SiC}$ results in a reduction of the number of domains in the Si layer by a factor of two. The experimental results shown here are in complete agreement with group theory expectations. The minimum number of domains in the $\mathrm{Si}$ layer on 3C-SiC APD-free layer is expected to be two. This highlights that the Si layer grown under these conditions reveals the minimum number of domains that can be expected on on-axis $3 \mathrm{C}-\mathrm{SiC} / \mathrm{Si}(001)$. To proceed to further domain annihilation, off-axis substrates are suggested to favor the growth of one of the two domains.

R.K. wishes to thank "Region Centre" and "Conseil General d'Indre et Loire" for their financial support. M.G. performed this work mostly as guest at CRHEA with support from Deutsche Forschungsgemeinschaft (Grant No. 1011/30-1).

${ }^{1}$ S. E. Saddow and A. Agarwal, Advances in Silicon Carbide Processing and Applications (Artech House, Norwood, MA, USA, 2004).

${ }^{2}$ S. E. Saddow, Silicon Carbide Biotechnology: A Biocompatible Semiconductor for Advanced Biomedical Devices and Applications (Elsevier Waltham, MA, 2012).

${ }^{3}$ S. Nishino, J. A. Powell, and A. H. Will, Appl. Phys. Lett. 42, 460 (1983).

${ }^{4}$ Y. T. Yang, K. L. Ekinci, X. M. H. Huang, L. M. Schiavone, M. L. Roukes, C. A. Zorman, and M. Mehregany, Appl. Phys. Lett. 78(2), 162 (2001).

${ }^{5}$ V. Cimalla, J. Pezoldt, and O. Ambacher, J. Phys. D: Appl. Phys. 40, 6386 (2007).

${ }^{6}$ M. P. Sarro, Sens. Actuators, A 82(1), 210 (2000).

${ }^{7}$ R. Boubekri, E. Cambril, L. Couraud, L. Bernardi, A. Madouri, M. Portail, T. Chassagne, C. Moisson, M. Zielinski, S. Jiao, J.-F. Michaud, D. Alquier, J. Bouloc, L. Nony, F. Bocquet, C. Loppacher, D. Martrou, and S. Gauthier, J. Appl. Phys. 116, 054304 (2014).

${ }^{8}$ S. Jiao, J. F. Michaud, M. Portail, A. Madouri, T. Chassagne, M. Zielinski, and D. Alquier, Mater. Lett. 77, 54 (2012).

${ }^{9}$ J. F. Michaud, M. Portail, T. Chassagne, M. Zielinski, and D. Alquier, Microelectron. Eng. 105, 65 (2013).

${ }^{10}$ R. Khazaka, E. Bahette, M. Portail, D. Alquier, and J. F. Michaud, Mater. Lett. 160, 28 (2015).

${ }^{11}$ J. F. Michaud, S. Jiao, A. E. Bazin, M. Portail, T. Chassagne, M. Zielinski, and D. Alquier, Mater. Res. Soc. Symp. Proc. 1246, B09-04 (2010).

${ }^{12}$ R. Anzalone, A. Severino, G. D'Arrigo, C. Bongiorno, G. Abbondanza, G. Foti, and F. La Via, J. Appl. Phys. 105, 084910 (2009).

${ }^{13}$ Y. Ishida, T. Takahashi, H. Okumura, K. Arai, and S. Yoshida, Chem. Vap. Deposition 12, 495 (2006).

${ }^{14}$ K. Shibahara, S. Nishino, and H. Matsunami, J. Cryst. Growth 78, 538 (1986).

${ }^{15}$ H. Matsunami, Physica B 185, 65 (1993).

${ }^{16}$ R. Khazaka, M. Portail, P. Vennéguès, D. Alquier, and J. F. Michaud, Acta Mater. 98, 336 (2015).

${ }^{17}$ L. O. Björketun, L. Hultman, O. Kordina, and J. E. Sundgren, J. Mater. Res. 13, 2632 (1998).

${ }^{18}$ R. Khazaka, M. Portail, P. Vennéguès, M. Zielinski, T. Chassagne, D. Alquier, and J. F. Michaud, Mater. Sci. Forum 821-823, 978 (2015).

${ }^{19}$ M. Grundmann, Phys. Status Solidi B 248, 805 (2011).

${ }^{20}$ M. Grundmann, T. Böntgen, and M. Lorenz, Phys. Rev. Lett. 105, 146102 (2010).

${ }^{21}$ See www.novasic.com/services/epitaxy/specs_3C-SiC-100.php for more information about the service of $3 \mathrm{C}-\mathrm{SiC}(100)$ epitaxy on silicon provided by NovaSiC

${ }^{22}$ T. Terzibaschian and R. Enderlein, Phys. Status Solidi B 133, 443 (1986).

${ }^{23}$ Y. Zhang, C. McAleese, H. Xiu, C. J. Humphreys, R. R. Lieten, B. Degroote, and G. Borghs, Appl. Phys. Lett. 91, 092125 (2007).

${ }^{24}$ Y. Ishida, T. Takahashi, H. Okumura, S. Yoshida, and T. Sekigawa, Jpn. J. Appl. Phys., Part 1 38, 3470 (1999). 